SUBJECT AREAS:

RISK FACTORS

METABOLIC SYNDROME

Received

7 July 2014

Accepted

16 February 2015

Published

30 March 2015

Correspondence and requests for materials should be addressed to

Y.L. (carol_liyang@

hotmail.com) or J.L. (jueli963258@126.

com)

* These authors contributed equally to this work.

\section{Using brachial-ankle pulse wave velocity to screen for metabolic syndrome in community populations}

\author{
Guanghua Wang ${ }^{1 *}$, Liang Zheng ${ }^{2 *}$, Xiankai Li ${ }^{3}$, Juanli Wu ${ }^{4}$, Lijuan Zhang ${ }^{4}$, Jie Zhang ${ }^{4}$, Liling Zou ${ }^{4}$, Xin Li ${ }^{2}$, \\ Yi Zhang ${ }^{3}$, Qian Zhou', Huimin Fan ${ }^{2}$, Yang Li ${ }^{5} \&$ Jue Li ${ }^{4}$
}

'Shanghai First Maternity and Infant Hospital, Tongii University School of Medicine, Shanghai, 201204, China, ${ }^{2}$ Research Center for Translational Medicine, Shanghai East Hospital, Tongii University School of Medicine, 200120, China, ${ }^{3}$ Department of Cardiology, Shanghai Tenth People's Hospital, Tongii University School of Medicine, Shanghai, 200072, China, ${ }^{4}$ Department of Prevention, Tongii University School of Medicine, Shanghai, 200092, China, ${ }^{5}$ Department of Health Management Medicine, Shanghai East Hospital, Tongji University School of Medicine, Shanghai, 200120, China.

The aim of this study is to investigate the viability of using brachial-ankle pulse wave velocity (baPWV) as a primary tool to screen metabolic syndrome (MetS), and to explore the risk factors of MetS in community populations. A total of 1914 subjects completed medical examination in Shanghai. BaPWV was significantly associated with the components of MetS. The area under curve (AUC) and its $95 \%$ confidence interval (CI) in total group were $62.50 \%$ and $60.00 \%-65.30 \%$ with the appropriate cut-off point being $1435 \mathrm{~cm} / \mathrm{sec}$. The AUC (95\%CI) of three subgroups (40-50 yrs, 50-60 yrs and over 60 yrs group) were $75.30 \%(67.48 \%-$ $83.35 \%), 63.35 \%(58.96 \%-67.60 \%), 55.37 \%(51.19 \%-60.01 \%)$, respectively. A clear pattern surfaced in the process of investigation: the younger were the subjects group, the better receiver operating characteristic (ROC) efficacy would emerge; and the higher sensitivity was, the better negative predictive value (NPV) would be. Male gender, high baPWV values, elevated uric acid (UA) and excess hypersensitive $\mathrm{C}$ reaction protein (hs-CRP) levels were stayed in the two regression models as the independent risk factors for MetS. We conclude that baPWV may serve as a potential screening tool for MetS at the cut-off point of $1435 \mathrm{~cm} /$ sec.

rachial-ankle pulse wave velocity (baPWV) is a promising yet relatively simple test that measures the stiffness of both aortic and peripheral arteries ${ }^{1,2}$ and a number of research groups propose using this technique to detect subclinical atherosclerotic changes. Many studies have demonstrated its reproducibility and validity ${ }^{3-5}$, with some investigators advocating that baPWV is not only an ideal marker for vascular damage $e^{6,7}$ but also a valuable predictor to the mortality resulted from cardiovascular diseases ${ }^{8}$. Due to the non-invasive nature and simplicity in performing all the measurements, baPWV has a potential screening value for vascular injuries in community populations.

MetS involves a combination of factors that increases the risk of cardiovascular diseases and type 2 diabetes, and its prevalence is increasing in many countries and in China alike. These risk factors include obesity, dysglycemia, raised blood pressure (BP), elevated triglyceride (TG) level, and low high-density lipoprotein cholesterol (HDL-C) level ${ }^{9}$. With the increasing prevalence of MetS in China, MetS has seriously burdened the society and, in particular, the families of those affected subjects as reported in literature ${ }^{10,11}$.

Early detection and treatment of MetS have been proven to reduce further cardiovascular disease morbidity and mortality ${ }^{12}$. However, with no immediate physical symptoms or medical problems associated with this disorder, the diagnosis of MetS remains challenging. The most widely recognized metabolic criteria (lipid profile, $\mathrm{BP}$ and glucose level) are commonly measured during hospitalization of patients ${ }^{13}$. The cost and availability of these diagnostic tests have hampered the mass screening in high-risk populations using above indices, especially in underdeveloped areas and developing countries. Hence, we launched this study to investigate the feasibility of using baPWV in screening for MetS in community populations. The current study focused on the efficacy of a non-invasive baPWV examination in screening individuals at high risk of MetS. Since it is well known that the incidence of MetS increases with age, we explored the screening capability of the method and appropriate cut-off point. 


\section{Methods}

Study population. Methods used in the present study were carried out in accordance with the approved guideline. At the same time, all experimental protocols were approved by the Ethics Committee of Tongji University, and all the participants signed informed consent.

Individuals were included if they met all the following criteria: (1) subjects with age 40 years and above; (2) subjects who were conventional residents in Lujiazui community, Shanghai; (3) individuals without hypertension crisis, multiple system organ failure, or other disease that may prevent them from measuring baPWV. Subjects were excluded if they did not meet above criteria. This community-based study adopted a cluster sampling scheme and randomly recruited a total of 2000 individuals between June 2009 and January 2010. Among these participants, 1914 subjects completed the lab tests and personal health and medical questionnaires. The response rate was $95.70 \%$

Anthropometric index and lab tests. Body mass index (BMI) was calculated as body weight in kilograms divided by the square of the body height in meters $\left(\mathrm{kg} / \mathrm{m}^{2}\right)$. The systolic and diastolic blood pressures (SBP and DBP, respectively) were obtained from the left arms of seated patients with an automatic BP monitor after 20 minutes of rest. Blood was drawn from the subjects in the morning after a 12-hour overnight fasting and was sent for analysis within four hours of collection. Biochemical markers such as uric acid (UA), total cholesterol (TC), TG, brain natriuretic peptide (BNP), hypersensitive $\mathrm{C}$ reaction protein (hs-CRP), homocyteine (Hcy), HDL-C, low density lipoprotein cholesterol (LDL-C), glycosylated hemoglobin Alc (HbAlc), and fasting plasma glucose (FPG) levels were analyzed using a biochemical auto analyzer in the Department of Clinical Laboratory, Shanghai East Hospital, Tongji University School of Medicine.

Lifestyle behaviors. In the present study, the term 'not married' referred to both unmarried and divorced individuals, 'low education level' meant less-than-12 years of full time education, and 'high education level' denoted equal to or greater than 12 years education. The subjects' current smoking status was derived from affirmative responses to the question "Are you a regular smoker now?" For participants reporting a history of smoking, those who had smoked within the past 6 months were defined as 'current smokers'; while those who had not smoked within the past 6 months as 'nonsmokers" ${ }^{14}$. Participants were also asked "How many alcoholic drinks do you have each week?" and "How many days each week do you usually drink alcohol?" These variables were used to determine the approximate number of alcoholic drinks consumed each day by the participant. A binary variable was constructed to distinguish between the participants consuming less than two and those drinking at least two alcoholic drinks every day.
Definition of MetS. The definition ${ }^{15,16}$ of MetS was as following:(1) Required criteria: BMI $\geq 25 \mathrm{~kg} / \mathrm{m}^{2}$ (waist circumference was not available in this study); (2) Plus any two of the following four factors: a. Elevated TG level: $\geq 1.7 \mathrm{mmol} / \mathrm{L}$ (or $150 \mathrm{mg} / \mathrm{dL}$ ); b. Reduced HDL-C level: $<40 \mathrm{mg} / \mathrm{dL}$ (male) or $50 \mathrm{mg} / \mathrm{dL}$ (female); c. Raised BP: SBP $\geq 130 \mathrm{mmHg}$ or DBP $\geq 85 \mathrm{mmHg}$, d. Elevated FPG level: $\geq 5.6 \mathrm{mmol} / \mathrm{L}$ (or $100 \mathrm{mg} / \mathrm{dL}$ ).

BaPWV measurement. A clinical device (baPWV-form) was used to automatically and simultaneously chronicle BP in both arms and ankles and document waves of the brachial and posterior tibial arteries using an automated oscillometric method ${ }^{17-19}$. BaPWV value can easily be checked through VP-1000 automated PWV/ABI analyzer (model VP-1000; Nippon Colin Ltd., Komaki, Japan). These values were measured after individuals had rested for at least 5 minutes. In case the values of baPWV obtained on the right and left sides were not identical ${ }^{20}$, we adopted the average value of left and right baPWV.

Multivariate model. The adjusted variables included in Model 1 were baPWV, gender, educational level, income level, marriage status, UA, Hs-CRP and BNP, as included in the Model 2 were baPWV, gender, educational level, income level, marriage status, smoking, alcohol drinking, UA, hs-CRP and BNP.

Statistical analysis. Continuous data were expressed as mean and standard deviation (SD). For the continuous variables, $t$-test was employed to analyze the difference between MetS and non-MetS groups. If the variances were not equal between two groups, nonparametric test was used instead of $t$-test. For the classified variables, a Chi-squared test $\left(\chi^{2}\right.$-test) was conducted to show the ratio difference between the two groups. Receiver operating characteristic (ROC) curve $e^{21,22}$ was constructed to determine the optimal baPWV threshold in order to discriminate MetS and nonMetS individuals. Afterwards, the baPWV value with the highest sum of sensitivity and specificity was identified as the cut-off point. A step-wise logistic regression analysis was conducted to explore the risk factors of MetS. All statistical analyses were performed using the SPSS17.0 (SPSS Inc., Chicago, IL, USA) software package. A twotailed $P$ value of $<0.05$ was considered statistically significant.

\section{Results}

Demographic characteristics and correlation between baPWV and the components of MetS. The ages of all subjects traverse from 40 to 89 years with mean age being $59.46 \pm 8.88$ years. As shown in Table 1, the subjects in MetS group exhibit higher mean age and BMI compared to those in non-MetS group $(P<0.01)$.

Table 1 | Comparison of demographic characteristics between MetS and Non-MetS group

\begin{tabular}{|c|c|c|c|c|}
\hline \multirow[b]{2}{*}{ Variables } & \multirow{2}{*}{$\begin{array}{l}\text { MetS group } \\
\text { mean } \pm S D\end{array}$} & \multirow{2}{*}{$\frac{\text { Non-MetS group }}{\text { mean } \pm S D}$} & \multirow[b]{2}{*}{$t / \chi^{2}$} & \multirow[b]{2}{*}{$P$} \\
\hline & & & & \\
\hline Age (years) & $60.7 \pm 8.9$ & $59.0 \pm 8.8$ & -3.58 & 0.001 \\
\hline $\mathrm{BMI}\left(\mathrm{kg} / \mathrm{m}^{2}\right)$ & $27.65 \pm 2.31$ & $22.95 \pm 2.66$ & -34.37 & 0.001 \\
\hline \multicolumn{5}{|l|}{ Gender [n(\%)] } \\
\hline Male & $175(35.64)$ & $444(31.20)$ & 3.28 & 0.069 \\
\hline Female & $316(64.36)$ & $979(68.80)$ & & \\
\hline \multicolumn{5}{|c|}{ Marital status [n(\%)] } \\
\hline Not married & $53(10.79)$ & $144(10.12)$ & 0.18 & 0.671 \\
\hline Married & $438(89.21)$ & $1279(89.88)$ & & \\
\hline \multicolumn{5}{|c|}{ Income(RMB per month) $[\mathrm{n}(\%)]$} \\
\hline Low & $436(88.80)$ & $1249(87.77)$ & 0.36 & 0.545 \\
\hline High & $55(11.20)$ & $174(12.23)$ & & \\
\hline \multicolumn{5}{|c|}{ Education Level [n(\%)] } \\
\hline Low & $409(83.30)$ & $1164(81.80)$ & 0.56 & 0.453 \\
\hline High & $82(16.70)$ & $259(18.20)$ & & \\
\hline \multicolumn{5}{|c|}{ History of diabetes [n(\%)] } \\
\hline Yes & $69(14.18)$ & $86(6.11)$ & 31.84 & 0.001 \\
\hline \multirow{2}{*}{\multicolumn{5}{|c|}{ Smoking [n(\%)] }} \\
\hline & & & & \\
\hline Yes & $372(75.76)$ & $1126(79.13)$ & 2.43 & 0.119 \\
\hline No & $119(24.24)$ & $297(20.87)$ & & \\
\hline \multicolumn{5}{|l|}{ Drinking $[n(\%)]$} \\
\hline Yes & $403(82.08)$ & $1232(86.58)$ & 5.93 & 0.015 \\
\hline No & $88(17.92)$ & $191(13.42)$ & & \\
\hline
\end{tabular}

Abbreviations: BMI, body mass index;

Marital status: not married refers to both unmarried and divorced individuals;

Income level: high level means greater than or equal to 5000 RMB per month;

Education level: low education level means full time education of less than 12 years, and high level means greater than or equal to 12 years. 
Table 2 | Comparison of biochemical characteristics between MetS and Non-MetS group

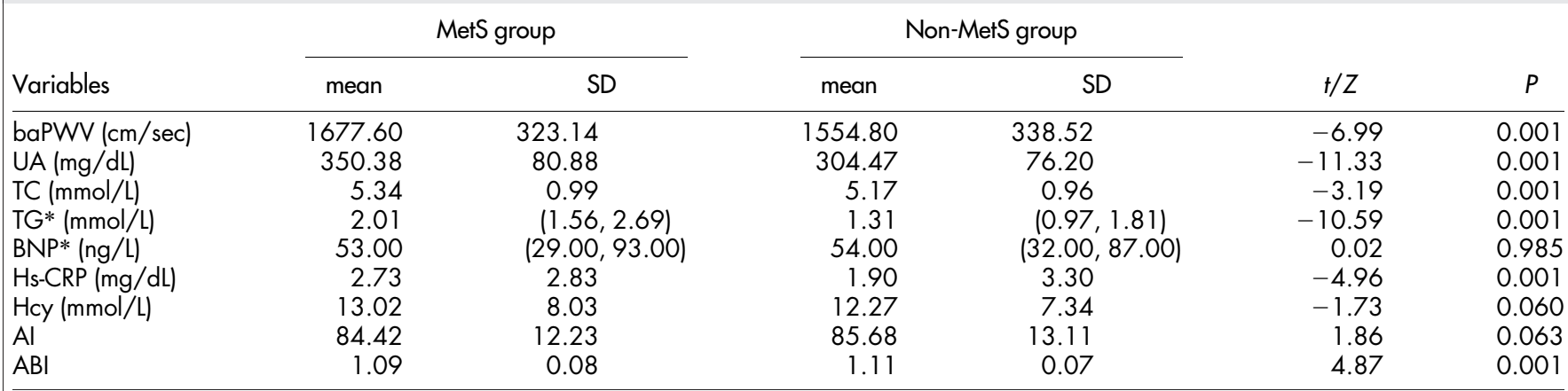

*Indicating the variances was not equal between two groups. Data was expressed as median and quartiles value;

Abbreviations: baPWV, brachial-ankle pulse wave velocity; UA, uric acid; TC, total cholesterol; TG, triglyceride; BNP, brain natriuretic peptide; Hs-CRP, hypersensitive C reaction protein; Hcy,

homocyteine; $\mathrm{Al}$, augmentation index; $\mathrm{ABI}$, ankle-brachial index.

\section{Table 3 | Correlation between baPWV and the components of MetS}

Variables

BMI $\left(\mathrm{kg} / \mathrm{m}^{2}\right)$

$\mathrm{SBP}(\mathrm{mm} \mathrm{Hg})$

$\mathrm{TG}(\mathrm{mmol} / \mathrm{L})$

HDL-C $(\mathrm{mmol} / \mathrm{L})$

LDL-C (mmol/L)

$\mathrm{HbAlc}(\%)$

Glucose (mmol/L)

$r$
0.11
0.60
0.14
0.09
0.05
0.19
0.24

Abbreviations: BMI, body mass index; SBP, systolic BP; TG, triglyceride; HDL-C, high density lipoprotein cholesterol; LDL-C, low density lipoprotein cholesterol; HbAlc, glycosylated hemoglobin Alc.

Other statistically significant differences have not been detected between the two groups of subjects, excepting the diabetes history and alcohol drinking status.

Our results demonstrate that there are significant differences between the two groups of subjects with nearly all the measured biochemical characteristics, excluding BNP, Hcy and augmentation index (AI) (Table 2). The subjects in the non-MetS group display a significantly higher ankle-brachial index (ABI) than those in the MetS group $(t=4.87, P<0.01)$.

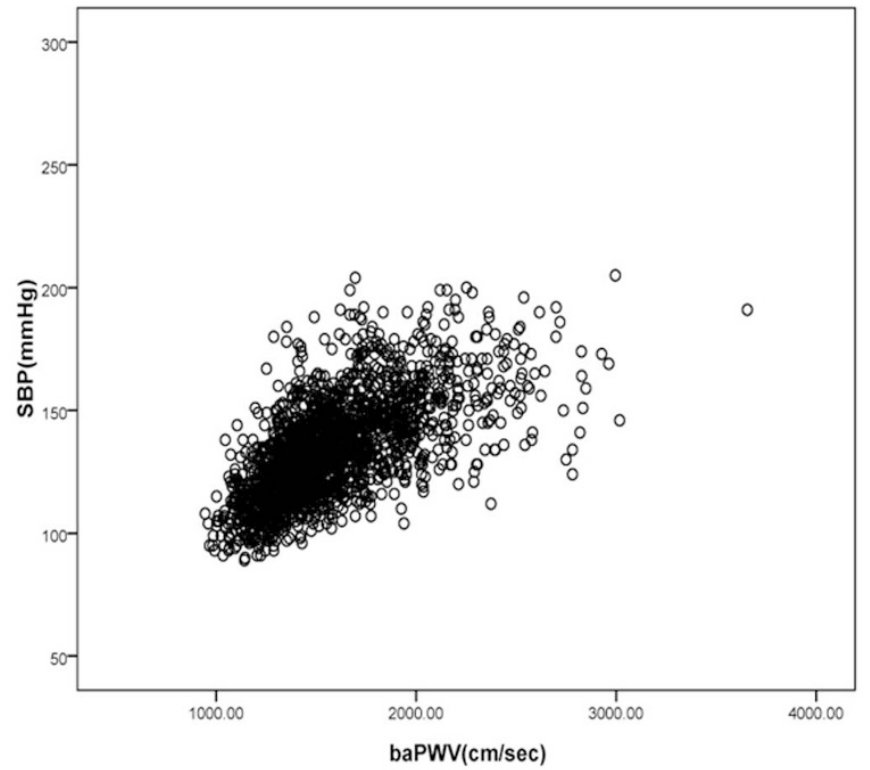

(a)
Conspicuously, the results in Table 3 prove a negative correlation between baPWV and HDL-C $(r=-0.09, P<0.01)$ and a positive relationship between baPWV and other variables in all subjects, while baPWV values illustrate a strong relationship with SBP, with the coefficient value being $0.60(P<0.01)$ (Figure 1$)$.

ROC curve and its cut-off point. Figure 2 presents the ROC curves in total group and subgroups (three age ranges). The AUC $(95 \% \mathrm{CI})$ in total group is $62.50 \%(60.00 \%-65.30 \%)$ with the appropriate

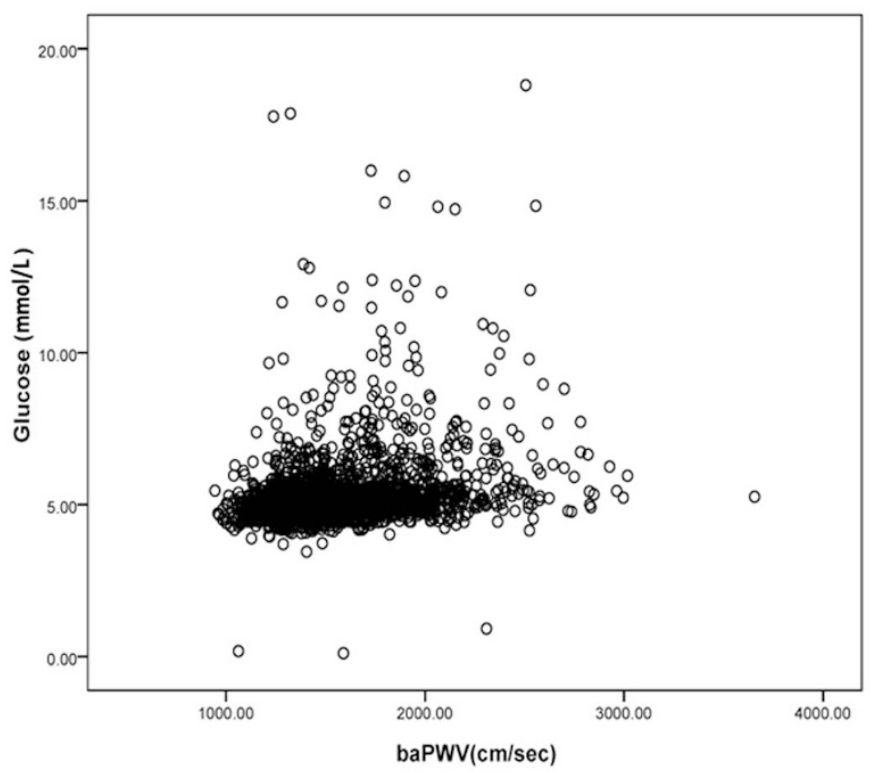

(b)

Figure $1 \mid$ Simple linear correlation between baPWV and other variables. (a) $\mathrm{r}=0.60, P=0.001$; (b) $\mathrm{r}=0.24, P=0.001$. 
cut-off point being $1435 \mathrm{~cm} / \mathrm{sec}$. The AUC in three subgroups (4050 yrs, 50-60 yrs and over 60 yrs group) are $75.30 \%, 63.35 \%$ and $55.37 \%$, respectively.

Table 4 displays the AUC, cut-off point, sensitivity, specificity, positive predictive value (PPV), negative predictive value (NPV) and Youden index in total group and all three subgroups. The baPWV at cut-off point of $1435 \mathrm{~cm} / \mathrm{sec}$ has resulted in the highest Youden index in total group, with a corresponding sensitivity being $76.06 \%$, specificity $50.43 \%$, PPV $33.84 \%$ and NPV $86.34 \%$. At the same time, its false negative rate is $23.94 \%$.

Risk factors of MetS in multiple logistic regression model. The MetS status is considered as a dependent variable, the same variables
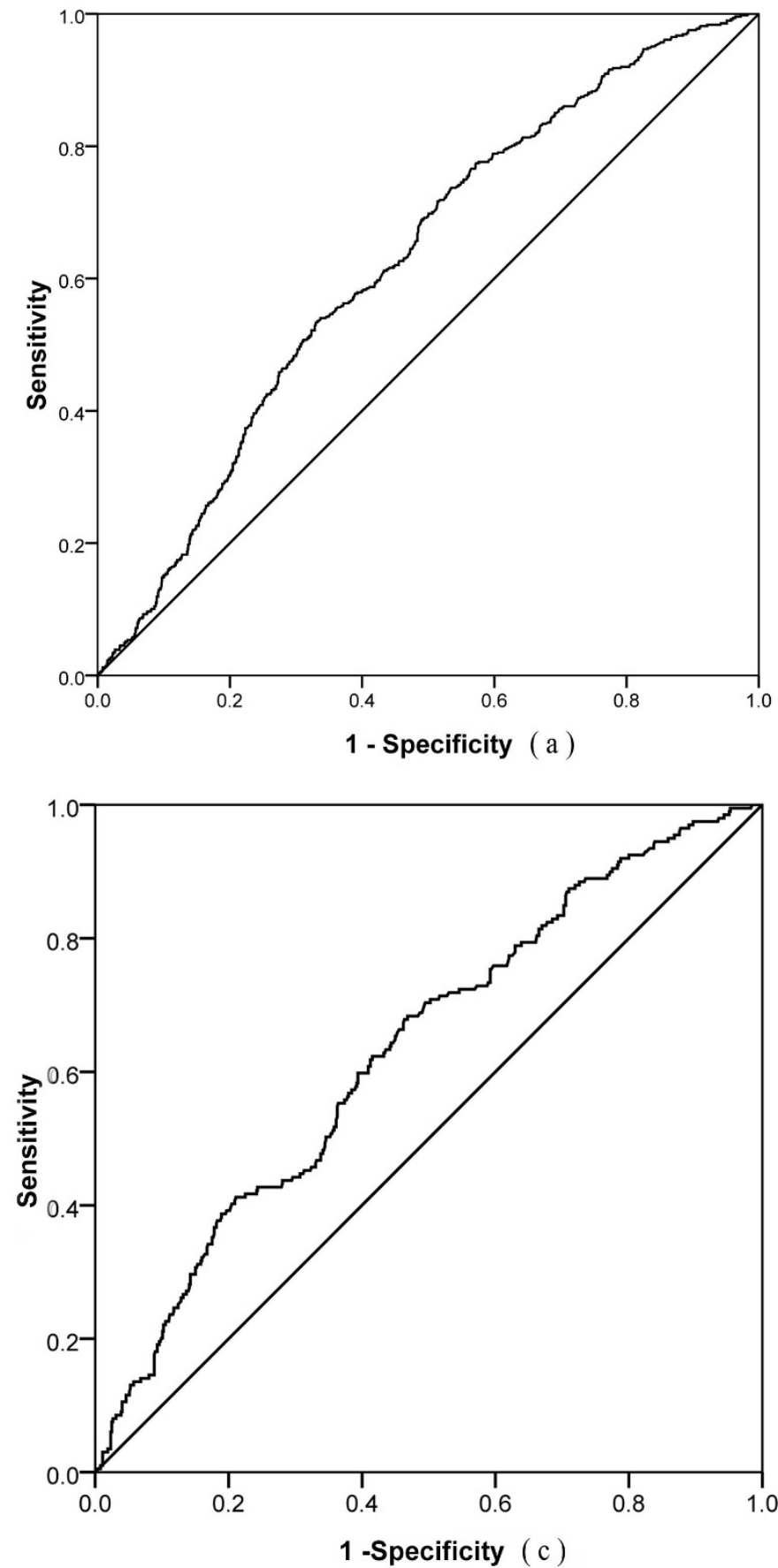

of gender, baPWV, UA and hs-CRP enter into two regression models (Model 1 and Model 2), the regression coefficients in Model 1 are $0.40,0.73,0.01$ and 0.06 , respectively; and the values turn into 0.45 $0.73,0.01$ and 0.06 , respectively, in Model 2 (Table 5). The Odds Ratio (OR) value (95\% CI) of baPWV is the highest in both models, being 2.08 (1.60-2.71) and 2.68 (1.60-2.71), respectively (Figure 3).

When baPWV data are divided into four factions of quartiles and the adjusted variables are same as those in Model 2, the OR and its $95 \% \mathrm{CI}$ are determined by logistic regression analysis (Table 6). Compared with the readings in lowest baPWV quartile, the adjusted OR (95\%CI) of having MetS in baPWV quartiles 2, 3, 4 are 1.60 (1.12-2.27), 1.63 (1.13-2.35), and $2.56(1.83-3.57)$ in the total group, respectively.
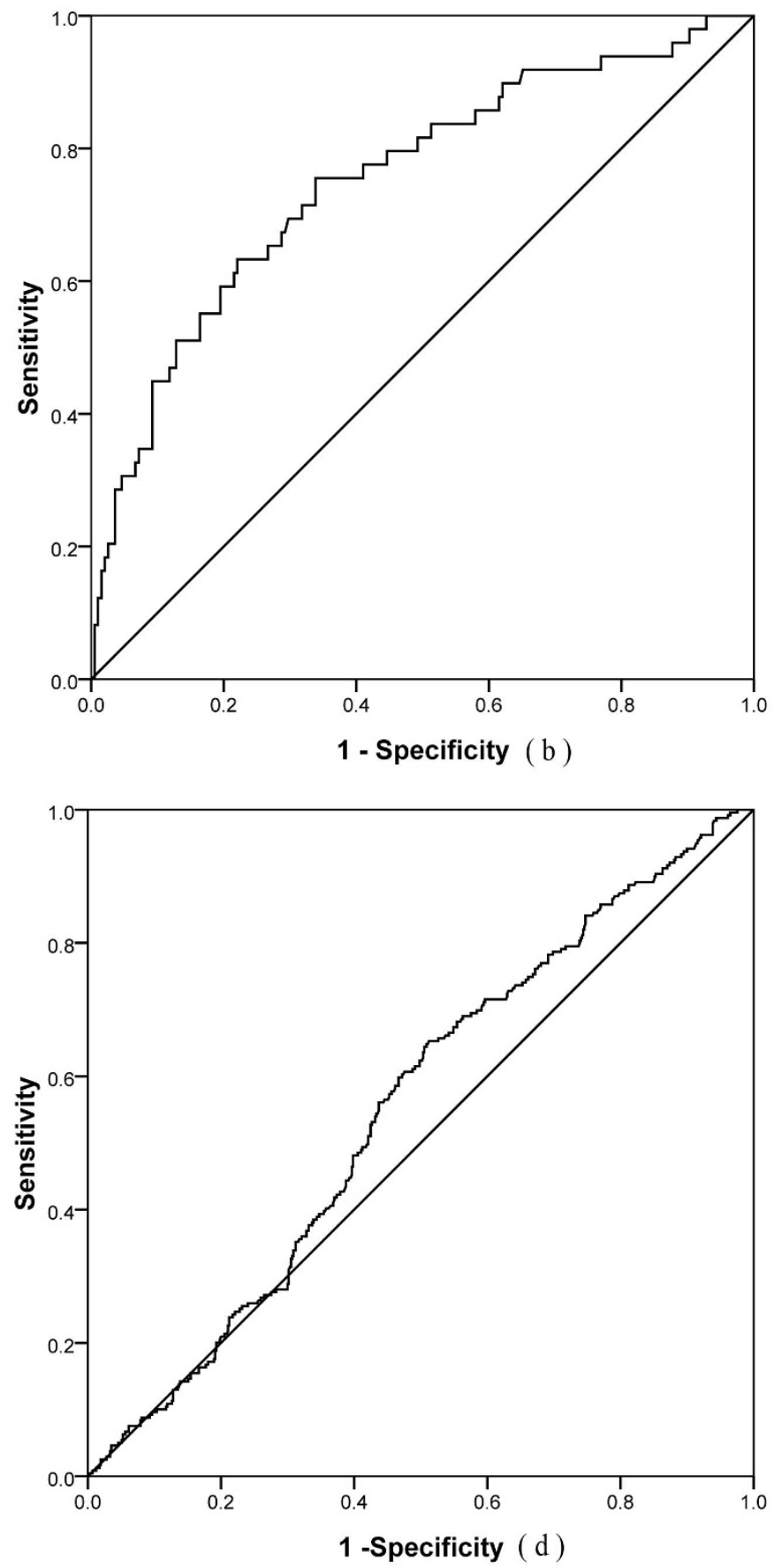

Figure $2 \mid$ ROC curve and AUC. (a) Total group: AUC $=62.50 \%$, 95\%CI of AUC: $60.00 \%-65.30 \%$, Cut-off point $=1435 \mathrm{~cm} / \mathrm{sec}$, Sensitivity $=76.06 \%$, Specificity $=50.43 \%$ (Limitation), Youden index $=0.26$; (b) $40-50$ yrs group: AUC $=75.30 \%, 95 \%$ CI: $67.48 \%-83.35 \%$; (c) 50-60 yrs group: AUC $=$ $63.35 \%$, 95\%CI: $58.96 \%-67.60 \%$; (d) $>60$ yrs group: AUC $=55.37 \%$, 95\%CI: $51.19 \%-60.01 \%$. 
Table 4 | Areas under the ROC curve, cut offs, sensitivity, specificity, PPV, NPV and Youden index of baPWV by age groups

\begin{tabular}{lcccccccc} 
Age & AUC (\%) (95\%Cl) & $P$ & Cut-off point & Sensitivity (\%) & Specificity (\%) & PPV (\%) & NPV (\%) & Youden index \\
\hline $40-50$ (yrs) & $75.30(67.48-83.35)$ & 0.001 & 1355 & 75.55 & 66.29 & 42.76 & 89.05 & 0.42 \\
$50-60$ (yrs) & $63.35(58.96-67.60)$ & 0.001 & 1427 & 68.35 & 53.28 & 32.78 & 83.47 & 0.22 \\
$>60$ (yrs) & $55.37(51.19-60.01)$ & 0.018 & 1680 & 65.33 & 48.87 & 30.03 & 80.87 & 0.14 \\
Total & $62.50(60.00-65.30)$ & 0.001 & 1435 & 76.06 & 50.43 & 33.84 & 86.34 & 0.26 \\
\hline
\end{tabular}

Abbreviations: AUC, areas under the ROC curve; PPV, positive predictive value; NPV, negative predictive value.

Table 5 | Risk factors and parameters of multiple logistic regression model

\begin{tabular}{|c|c|c|c|c|c|c|}
\hline Variables & $\beta^{d}$ & SE & Wald & $P$ & OR & $95 \% \mathrm{Cl}$ \\
\hline \multicolumn{7}{|l|}{ Model 1"a } \\
\hline Gender & 0.40 & 0.13 & 9.04 & 0.001 & 1.49 & $(1.15-1.93)$ \\
\hline $\mathrm{BaPWV}^{\mathrm{b}}$ & 0.73 & 0.13 & 30.15 & 0.001 & 2.08 & $(1.60-2.71)$ \\
\hline UA & 0.01 & 0.01 & 92.23 & 0.001 & 1.02 & $(1.01-1.03)$ \\
\hline $\begin{array}{l}\text { Hs-CRP } \\
\text { Model } 2^{c}\end{array}$ & 0.06 & 0.01 & 12.92 & 0.001 & 1.06 & $(1.03-1.10)$ \\
\hline \multicolumn{7}{|l|}{ Model2c } \\
\hline Gender & 0.45 & 0.17 & 20.91 & 0.001 & 1.58 & $(1.11-2.23)$ \\
\hline $\mathrm{BaPWV}^{\mathrm{b}}$ & 0.73 & 0.13 & 30.24 & 0.001 & 2.68 & $(1.60-2.71)$ \\
\hline UA & 0.01 & 0.00 & 90.69 & 0.001 & 1.01 & $(1.01-1.02)$ \\
\hline Hs-CRP & 0.06 & 0.01 & 12.89 & 0.001 & 1.07 & $(1.03-1.11)$ \\
\hline
\end{tabular}

aAdjusted variables in model 1: baPWV, gender (male vs. female), educational level, income level, marriage status, UA, Hs-CRP and BNP;

bBaPWV abnormal range: $\geq 1435 \mathrm{~cm} / \mathrm{sec}$, normal range, $<1435 \mathrm{~cm} / \mathrm{sec} ;$

'Adjusted variables in model2: baPWV, gender, educational level, income level, marriage status, smoking, alcohol drinking, UA, hs-CRP and BNP,

Regression coefficient.

\section{Discussion}

With the change of living rhythm and dietary style in China, the prevalence of MetS has drastically increased in the last decade throughout the Country. Using the International Diabetes Federation (IDF) definition, $\mathrm{Hu} \mathrm{XS}$ et $\mathrm{al}^{23}$ reported that the prevalence of MetS had been 17.84\% in 2005 in Jiangsu Province. In 2010, research carried out by Shao YQ et $\mathrm{al}^{24}$ indicated that the prevalence of MetS had been $19.21 \%$ in Shanghai area derived from using the same definition. Yet the society lacks a convenient, cost-efficient method to screen for the high-risk MetS individuals in communities.

Brachial-ankle pulse wave velocity (baPWV) is a newly-emerged technique that is capable of evaluating arterial stiffness in a relatively swift and economic way, predicting the cardiovascular risk factors in patients. The subjects with arteriosclerosis share some common pathology and clinical manifestations with subjects suffering MetS; and the existence of a strong association between MetS and arterial stiffness has been demonstrated in many studies ${ }^{25-27}$. In addition, a number of reports have verified that the mechanisms underlying the association include, but not limited to, the common inflammatory pre-cursors and oxidative stress appearance ${ }^{28}$. The simultaneous occurrence of MetS and proinflammatory and oxidative stress markers increases the likelihood of arterial stiffness ${ }^{29}$. Since the baPWV measurements increase before atherosclerosis ${ }^{30}$, we assume that baPWV is likely to be a potential screening tool for MetS.

Our results in this report show that baPWV values correlate with all components of MetS, substantiating the outcomes reported by Zhao WW et $\mathrm{al}^{30}$. In general, our data suggest that the prevalence of MetS is $25.65 \%$ in the middle-aged and elderly populations in Shanghai area. Although there are some differences in selecting participants with the above studies, our results are consistent with theirs, and the increasing prevalence of MetS in the Chinese population is unquestionably plain.

Thus far, there is not a generally accepted cut-off point of baPWV in screening individuals at risk of MetS. In this study, the discriminatory value of baPWV measurements for MetS has been established in the community population-based study with 1914

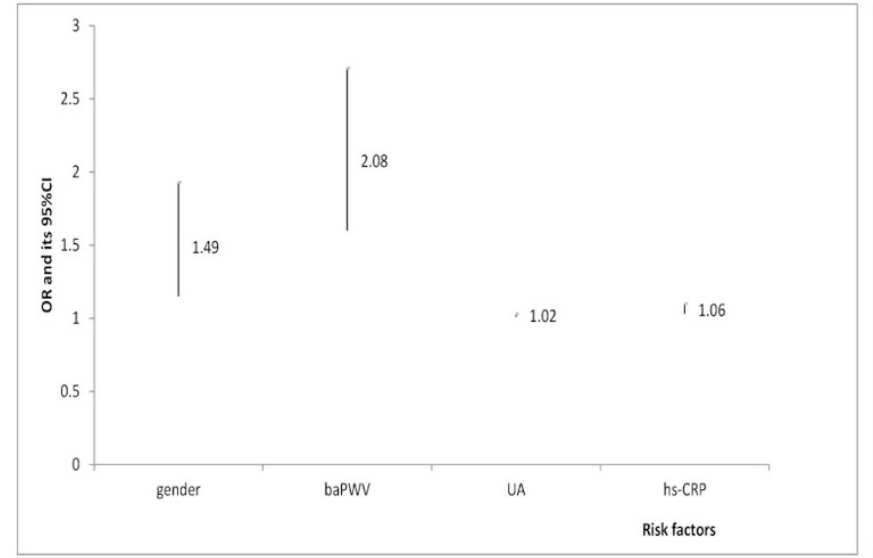

(a)

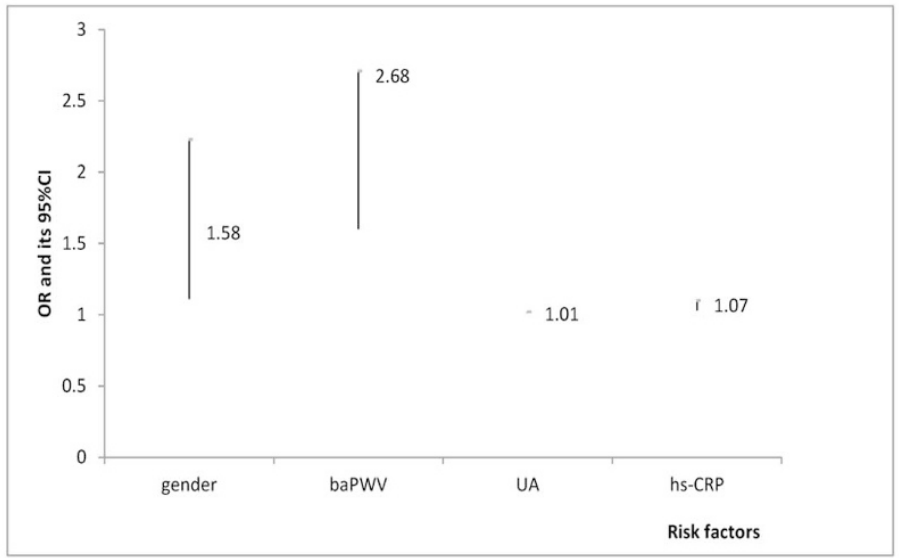

(b)

Figure $3 \mid$ OR value and its 95\%CI in different models. (a) Model 1; (b) Model 2. 
Table $6 \mid$ Odds ratio and $95 \% \mathrm{Cl}$ of baPWV for MetS in regression model

\begin{tabular}{|c|c|c|c|c|c|c|}
\hline Quartile & \multicolumn{2}{|c|}{ Male } & \multicolumn{2}{|c|}{ Female } & \multicolumn{2}{|c|}{ Total } \\
\hline Q1 & 1.00 & Reference & 1.00 & Reference & 1.00 & Reference \\
\hline Q3 & 1.66 & $0.89-3.10$ & 1.52 & $0.95-2.41$ & 1.63 & $1.13-2.35$ \\
\hline Q4 & 2.76 & $1.55-4.92$ & 2.25 & $1.48-3.42$ & 2.56 & $1.83-3.57$ \\
\hline
\end{tabular}

Adjusted for baPWV, gender, educational level, income level, marriage status, smoking, alcohol drinking, UA, hs-CRP and BNP; The baPWV was divided into four groups by quartile (<1378, 1378-1569, $1569-1818, \geq 1818 \mathrm{~cm} / \mathrm{sec}$ in men; $<1324,1324-1490,1490-1738, \geq 1738 \mathrm{~cm} / \mathrm{sec}$ in women; $<1336,1336-1516,1516-1704, \geq 1704 \mathrm{~cm} / \mathrm{sec} \mathrm{in} \mathrm{total).}$

subjects. More importantly, we have determined an optimal cut-off point of baPWV $(1435 \mathrm{~cm} / \mathrm{sec})$, which could be a potential index variable to screen for high risk individuals of MetS in local Chinese populations.

Moreover, besides establishing the useful cut-off point of baPWV, we have calculated baPWV quartiles in logistic model as well. With the increase of baPWV in quartiles, the OR of baPWV for MetS boosts as well, confirming the similar results of an earlier study ${ }^{31}$. The difference between two genders has already been reported in our previous publication $^{32}$.

The ROC curve attained from our results has demonstrated that baPWV may be considered as a screening index to discriminate between those with and without MetS. In total group, the AUC $(95 \% \mathrm{CI})$ is $62.50 \%(60.00 \%-65.30 \%)$ and the optimal cut-off point of baPWV for MetS is $1435 \mathrm{~cm} / \mathrm{sec}$, which is higher than Yamashina's suggestion $^{20}$, but lower than Lin WY's implication ${ }^{31}$. It is noteworthy that the false negative rate $(23.94 \%)$ is lower than the previous reports, meaning omission of a diagnostic index is unlikely. In addition, it is interesting to find that the younger a subject's age is, the better ROC efficacy he/she acquires; the higher sensitivity is, the better NPV surfaces. Similarly, the older age is, the higher baPWV appears; the lower specificity is, the lower PPV emerges. This trend is consistent with the results reported by Wang $\mathrm{F}^{33}$, who has tried to predict the MetS with BMI. However, our AUC outcome in different subgroups is better than that of the previous report $^{33}$, supporting the notion that baPWV has a better capability for screening MetS than BMI, waist circumference(WC) or waist to hip ratio (WHR).

Analysis of multiple logistic regressions demonstrates that gender, baPWV, UA and hs-CRP are the high risk factors of MetS, consistent with the results of other reports ${ }^{29,34,35}$. Individuals with higher baPWV are twice more likely to have MetS compared to those with lower baPWV. Hyperuricemia is another significant predictor of $\mathrm{MetS}^{36}$, and chronic low-grade inflammation in MetS is an underlying mechanism mediated in part by the pro-inflammatory properties of $\mathrm{UA}^{37,38}$ and may trigger the elevated baPWV. UA has been found to play two roles in promoting inflammation: as a monosodium urate crystal and as a soluble factor ${ }^{34}$. Inflammation (assessed by hs-CRP) may cling to a possible, underlying relationship between baPWV and MetS $^{31,39}$.

It should be pointed out that sensitivity of this screening scheme may be underestimated. Since obesity is an essential diagnostic criterion for MetS and the condition may lead to other metabolic disorders $^{40,41}$, a possible limitation exists as we adopt BMI as a surrogate variable for waist circumference in defining people with central obesity $^{42}$. The fact that most Asians rather have normal BMI and high WHR (central obesity) may cause a high false negative rate.

Besides, there are several limitations in the study. Firstly, the study participants consist of middle-aged and elderly individuals, and the AUC is likely underestimated, since the younger age the subgroup members are of, the better ROC efficacy turns up. Secondly, the specificity may not be optimal and most advantageous, and further studies are warranted to verify this. Thirdly, we cannot prove the causality between baPWV and MetS since this is a cross-section study. However, we have found a close association between baPWV and MetS as well as the MetS components, which are also risk factors of cardiovascular diseases. Prospective studies are needed to establish the time sequence in the relationship between UA, hs$\mathrm{CRP}$, baPWV, and MetS, and to fine tune and improve the screening procedures.

In conclusion, baPWV is a potential index variable to screen high risk individuals of MetS and we would need further work to test the utility as a screening tool. Moreover, a higher baPWV, hs-CRP, UA level, as well as male gender are the independent risk factors for MetS.

1. Yamashina, A. et al. Validity, reproducibility, and clinical significance of noninvasive brachial-ankle pulse wave velocity measurement. Hypertens. Res. 25, 359-364 (2002).

2. Tomiyama, H. et al. Influences of age and gender on results of noninvasive brachial-ankle pulse wave velocity measurement--a survey of 12517 subjects. Atherosclerosis 166, 303-309 (2003).

3. Figueroa, A. et al. Effects of diet and/or low-intensity resistance exercise training on arterial stiffness, adiposity, and lean mass in obese postmenopausal women. Am. J. Hypertens. 26, 416-423 (2013).

4. Nakamura, M. et al. Brachial-ankle pulse wave velocity as a risk stratification index for the short-term prognosis of type 2 diabetic patients with coronary artery disease. Hypertens. Res. 33, 1018-1024 (2010).

5. Chen, S. C. et al. Brachial-ankle pulse wave velocity and rate of renal function decline and mortality in chronic kidney disease. Clin. J. Am. Soc. Nephrol. 6, 724-732 (2011).

6. Hsu, S. R. et al. Risk factors of accelerated progression of peripheral artery disease in hemodialysis. Kaohsiung J. Med. Sci. 29, 82-87 (2013).

7. Doba, N. et al. Changes in ankle brachial pulse wave velocity during a five-year follow-up period in older Japanese adults: sub-analysis results of the health research volunteer study in Japan. Intern. Med. 52, 21-27 (2013).

8. Lehmann, E. D., Riley, W. A., Clarkson, P. \& Gosling, R. G. Non-invasive assessment of cardiovascular disease in diabetes mellitus. Lancet 350 Suppl 1, SI14-19 (1997).

9. Alberti, K. G. et al. Harmonizing the metabolic syndrome: a joint interim statement of the International Diabetes Federation Task Force on Epidemiology and Prevention; National Heart, Lung, and Blood Institute; American Heart Association; World Heart Federation; International Atherosclerosis Society; and International Association for the Study of Obesity. Circulation 120, 1640-1645 (2009).

10. Wu, S. et al. Association between erythrocyte parameters and metabolic syndrome in urban Han Chinese: a longitudinal cohort study. BMC Public Health 13, 989 (2013).

11. Workalemahu, T., Gelaye, B., Berhane, Y. \& Williams, M. A. Physical activity and metabolic syndrome among Ethiopian adults. Am. J. Hypertens. 26, 535-540 (2013).

12. Grundy, S. M. et al. Diagnosis and management of the metabolic syndrome: an American Heart Association/National Heart, Lung, and Blood Institute Scientific Statement. Circulation 112, 2735-2752 (2005).

13. Patel, P. V., Gilski, D. \& Morrison, J. Using waist circumference to screen for metabolic syndrome in an inpatient population. Crit Pathw Cardiol 9, 152-155 (2010).

14. Tuomilehto, J. et al. Urinary sodium excretion and cardiovascular mortality in Finland: a prospective study. Lancet 357, 848-851 (2001).

15. Sabo, R. T. et al. Parental and offspring associations of the metabolic syndrome in the Fels Longitudinal Study. Am. J. Clin. Nutr. 96, 461-466 (2012).

16. Alberti, K. G., Zimmet, P. \& Shaw, J. The metabolic syndrome--a new worldwide definition. Lancet 366, 1059-1062 (2005).

17. $\mathrm{Su}, \mathrm{H}$. M. et al. Impact of systolic time intervals on the relationship between arterial stiffness and left ventricular hypertrophy. Atherosclerosis 223, 171-176 (2012). 
18. Chen, S. C., Chang, J. M., Tsai, Y. C., Su, H. M. \& Chen, H. C. Brachial-ankle pulse wave velocity and brachial pre-ejection period to ejection time ratio with renal outcomes in chronic kidney disease. Hypertens. Res. 35, 1159-1163 (2012).

19. $\mathrm{Su}, \mathrm{H}$. M. et al. Association of brachial-ankle pulse wave velocity, ankle-brachial index and ratio of brachial pre-ejection period to ejection time with left ventricular hypertrophy. Am. J. Med. Sci. 347, 289-294 (2014).

20. Yamashina, A. et al. Brachial-ankle pulse wave velocity as a marker of atherosclerotic vascular damage and cardiovascular risk. Hypertens. Res. 26, 615-622 (2003).

21. Guo, X. et al. Sensitivity and specificity of ankle-brachial index for detecting angiographic stenosis of peripheral arteries. Circ. J. 72, 605-610 (2008).

22. Park, K. H. et al. Clinical significance of framingham risk score, flow-mediated dilation and pulse wave velocity in patients with stable angina. Circ. J. 75, 1177-1183 (2011).

23. Hu, X. S. et al. [Study on the prevalence of metabolic syndrome among 35-74 yearolds in Jiangsu province]. Chin J Epidemiol 27, 751-756 (2006).

24. Shao, Y. Q. et al. Epidemiologyical features of metabolic syndrome in 35-74 yearold residents in suburban areas of Shanghai. Chinese general practice 14, 2069-2071 (2011)

25. Tok, D. et al. Increased epicardial fat thickness is associated with low grade systemic inflammation in metabolic syndrome. Turk Kardiyol Dern Ars 40 , 690-695 (2012).

26. Satoh, H., Kishi, R. \& Tsutsui, H. Metabolic syndrome is a significant and independent risk factor for increased arterial stiffness in Japanese subjects. Hypertens. Res. 32, 1067-1071 (2009).

27. Li, C. I. et al. Metabolic syndrome is associated with change in subclinical arterial stiffness: a community-based Taichung community health study. BMC Public Health 11, 808 (2011).

28. Zhang, Y. et al. Inflammation and oxidative stress are associated with the prevalence of high aankle-brachial index in metabolic syndrome patients without chronic renal failure. Int. J. Med. Sci. 10, 183-190 (2013).

29. Weng, C. et al. Age- and gender dependent association between components of metabolic syndrome and subclinical arterial stiffness in a Chinese population. Int. J. Med. Sci. 9, 730-737 (2012).

30. Zhao, W. W. et al. Serum high-density lipoprotein cholesterol and progression to arterial stiffness in middle-aged and elderly Chinese. Nutr. Metab. Carbiovasc. Dis. 23, 973-979 (2013).

31. Lin, W. Y. et al. In addition to insulin resistance and obesity, brachial-ankle pulse wave velocity is strongly associated with metabolic syndrome in Chinese--a population-based study (Taichung Community Health Study, TCHS). J. Atheroscler. Thromb. 16, 105-112 (2009).

32. Li, X. et al. Can we early diagnose metabolic syndrome using brachial-ankle pulse wave velocity in community population? Chin Med J (Engl) 127, 3116-3120 (2014).

33. Wang, F. et al. Waist circumference, body mass index and waist to hip ratio for prediction of the metabolic syndrome in Chinese. Nutr. Metab. Carbiovasc. Dis. 19, 542-547 (2009).

34. Sun, N., Zhang, Y., Tian, J. L. \& Wang, H. Relationship between uric acid and arterial stiffness in the elderly with metabolic syndrome components. Chin Med J (Engl) 126, 3097-3102 (2013).
35. Deetman, P. E., Bakker, S. J. \& Dullaart, R. P. High sensitive C-reactive protein and serum amyloid A are inversely related to serum bilirubin: effect-modification by metabolic syndrome. Cardiovasc. Diabetol. 12, 166 (2013).

36. Nagahama, K. et al. Hyperuricemia predicts future metabolic syndrome: a 4-year follow-up study of a large screened cohort in Okinawa, Japan. Hypertens. Res. 37, 232-238 (2013)

37. Moller, D. E. \& Kaufman, K. D. Metabolic syndrome: a clinical and molecular perspective. Annu. Rev. Med. 56, 45-62 (2005).

38. Grundy, S. M. et al. Diagnosis and management of the metabolic syndrome: an American Heart Association/National Heart, Lung, and Blood Institute scientific statement: Executive Summary. Annu. Rev. Med. 4, 198-203 (2005).

39. Jha, H. C., Divya, A., Prasad, J. \& Mittal, A. Plasma circulatory markers in male and female patients with coronary artery disease. Heart Lung 39, 296-303 (2010).

40. Purkayastha, S. \& Cai, D. Neuroinflammatory basis of metabolic syndrome. Mol Metab 2, 356-363 (2013).

41. Despres, J. P. \& Lemieux, I. Abdominal obesity and metabolic syndrome. Nature 444, 881-887 (2006).

42. Turati, F. et al. Metabolic syndrome and hepatocellular carcinoma risk. Br. J. Cancer 108, 222-228 (2013).

\section{Acknowledgments}

This study was supported by grants from the National Nature Science Foundation of China (No. 81170325); International Science \& Technology Cooperation Program of China (No. 2011DFB30010); National Nature Science Foundation of China (No. 81102203); Fundamental Research Funds for the Central Universities (Study on the key technology of disaster warning and relief for rural remote areas. National Key Basic Research Program of China (No. 2013CB531100).

\section{Author contributions}

J.L. and Y.L. proposed the conceptual idea and provided financial support through grant application. G.W. and L.Z. designed and carried out the study, and analyzed the data and wrote the manuscript. X.L., J.W., L.Z., J.Z., L.Z., X.L., Y.Z., Q.Z. and H.F. participated in discussing the results and in writing the manuscript. All authors reviewed the manuscript.

\section{Additional information}

Competing financial interests: The authors declare no competing financial interests.

How to cite this article: Wang, G. et al. Using brachial-ankle pulse wave velocity to screen for metabolic syndrome in community populations. Sci. Rep. 5, 9438; DOI:10.1038/ srep09438 (2015).

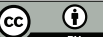

This work is licensed under a Creative Commons Attribution 4.0 International License. The images or other third party material in this article are included in the article's Creative Commons license, unless indicated otherwise in the credit line; if the material is not included under the Creative Commons license, users will need to obtain permission from the license holder in order to reproduce the material. To view a copy of this license, visit http://creativecommons.org/licenses/by/4.0/ 